\title{
Signal responsive transient coacervation in complex coacervate core micelles
}

Reece W. Lewis, Benjamin Klemm, Mariano Macchione, Rienk Eelkema*

Department of Chemical Engineering, Delft University of Technology, Van der Maasweg 9, 2629 HZ Delft, The Netherlands.

* Correspondence to: R.Eelkema@ tudelft.nl 


\begin{abstract}
Triggered coacervate phase (de)stabilisation in complex coacervate core micelles (C3Ms) has traditionally been limited to changes in $\mathrm{pH}$ and salt concentration, limiting options in responsive $\mathrm{C} 3 \mathrm{M}$ material design. To expand this toolbox, we have developed $\mathrm{C} 3 \mathrm{Ms}$, that, at constant physiological $\mathrm{pH}$, assemble and disassemble by coupling to a chemical reaction network $(\mathrm{CRN})$ driven by the conversion of electron deficient allyl acetates and thiol or amine nucleophiles. This CRN produces transient quaternization of tertiary amine-functionalised block copolymers, which can then form the complex coacervate phase. We demonstrate triggered C3M assembly using two different allyl acetates, resulting in dramatically different assembly rates from hours to days. These are applied in various combinations with selected nucleophiles, demonstrating sequential signal induced $\mathrm{C} 3 \mathrm{M}$ formation and deformation, as well as transient non-equilibrium (de)formation. We expect that timed and signal-responsive control over coacervate phase formation at physiological $\mathrm{pH}$ will find application in nucleic acid delivery, nano reactors and protocell research.
\end{abstract}




\section{Introduction}

Dynamic control over assembly and disassembly allows for materials which display intelligent responses to changes in their environment, with application in diagnostics, ${ }^{1}$ artificial protocell research, ${ }^{2}$ self-healing and drug release to name a few. ${ }^{3-5}$ Many synthetic materials have been designed to switch between equilibrium states upon reaction with a desired signal, for example micelles transitioning from assembled to disassembled states for site specific cargo release. ${ }^{6}$ In contrast to this, nature often utilises fuel-driven processes which achieve non-equilibrium structures with features including spatiotemporal control, responsiveness and autoconfiguration. These features allow for complex cellular functions such as division, motility and intracellular transport. ${ }^{7,8}$ Synthetic non-equilibrium assemblies can be attained from building blocks which are incorporated into a chemical reaction network (CRN). Here, non-assembling precursors are converted to an assembling product by irreversible reaction with a chemical reagent, the fuel. A second deactivating reaction later converts the product back to its non-assembling state. Thus, assembly only occurs where and when fuel is available, allowing for spatiotemporal control without an external change to the environment. ${ }^{9}$

In this work we describe a new approach for both signal induced and fuel-driven coacervation of polyelectrolytes and explore its application in programmed micelle (dis)assembly. Coacervation occurs during the mixing of oppositely charged polyelectrolytes in aqueous solution, leading to the formation of a water-insoluble macromolecule rich phase (the complex coacervate) and a diluted bulk phase. ${ }^{10,11}$ This process is initiated by coulombic attraction between the macroions and primarily driven by an entropy increase due to counterion release from the electrical double layer into the bulk solution. ${ }^{12,13}$ When one (or more) water-soluble neutral block(s) are attached to the polyelectrolytes, complex coacervate core micelles (C3Ms) (also known as polyion complex micelles) can be formed. ${ }^{14}$ The resultant core-shell structures have a water-insoluble (but hydrated) coacervate core, surrounded by a stabilising water- 
soluble shell. The relatively hydrophilic core of C3Ms allows for incorporation and protection of hydrophilic substrates such as enzymes and nucleic acids, which is often not possible for conventional amphiphilic micelles. ${ }^{15-19}$

Control over micelle formation and disassembly in $\mathrm{C} 3 \mathrm{Ms}$ is most directly achieved by controlling assembly of the coacervate core. Coacervates are most favoured to form at low salt concentrations from polyelectrolytes with large, fully ionized core blocks, combined at an equimolar ratio of cationic to anionic monomers. ${ }^{20,21}$ This causes inherent $\mathrm{pH}$ (for weak polyelectrolytes) and salt responsiveness in C3Ms, inspiring the design of various stimuli responsive gels, encapsulated catalyst and drug delivery systems. ${ }^{22-27} \mathrm{C} 3 \mathrm{Ms}$ able to reversibly assemble at constant $\mathrm{pH}$ and salt concentration are, however, hardly reported and can be considered advantageous as they avoid issues arising from salt and buffer accumulation as well as operation in $\mathrm{pH}$ sensitive environments. Conceptually two approaches for this can be envisaged. The first approach involves inducing a reduction in molecular weight for one of the polyelectrolytes. This method was applied in an elaborate $\mathrm{C} 3 \mathrm{M}$ design whereby anionic coordination complexes, themselves of insufficient charge to form micelles with a cationic block-copolymer, were crosslinked by a disulfide bond containing linker. The crosslinks resulted in micelle formation, which could be transiently reversed by addition of a reducing agent (dithiothreitol) to cleave the disulfide bonds, reducing the molecular weight of the anionic species and breaking apart the micelles. ${ }^{28}$ It is of note that similar polyelectrolyte cleavage approaches have been demonstrated in reversible coacervate droplets (non-micelle forming), which have been widely investigated as models for membraneless organelles and protocells. ${ }^{29-32}$

A second approach would be to change the ionisation or net charge of the polymer, which (at constant $\mathrm{pH}$ ) has only been reported for coacervate droplets. ${ }^{33,34}$ For example Donau et al. recently reported a fuel-driven system operating at $\mathrm{pH}$ 5.3, whereby an aspartate functionalised 
peptide is ring closed to the anhydride after addition of EDC (fuel), causing a change in peptide net charge from +1 to +3 . This change in charge was sufficient to trigger the formation of a coacervate phase with RNA and, since the anhydride is unstable and hydrolyses back to the aspartate, the coacervate phase formation is transient. ${ }^{34}$

Our group has recently reported a new CRN fuelled by electron deficient allyl acetates, which when reacted with tertiary amines yield a quaternary cationic adduct. Reaction of this adduct with a competing nucleophile is then able to regenerate the starting tertiary amine, completing the cycle. ${ }^{35}$ The allyl acetate fuel is essentially an activated Michael acceptor, which have been shown to have reactivities spanning many orders of magnitude for triggered release of good leaving groups (such as tertiary amines). ${ }^{36}$ In this work, we demonstrate the utility of the allyl acetate CRN to achieve reversible unimer to $\mathrm{C} 3 \mathrm{M}$ transitions by transient quaternization of tertiary amine functionalised polymers mixed with a polyanion. By tuning the rates of the ionization and deionization reactions, both signal induced and transient fuel-driven micelle (dis)assemblies are accessible (Scheme 1). 


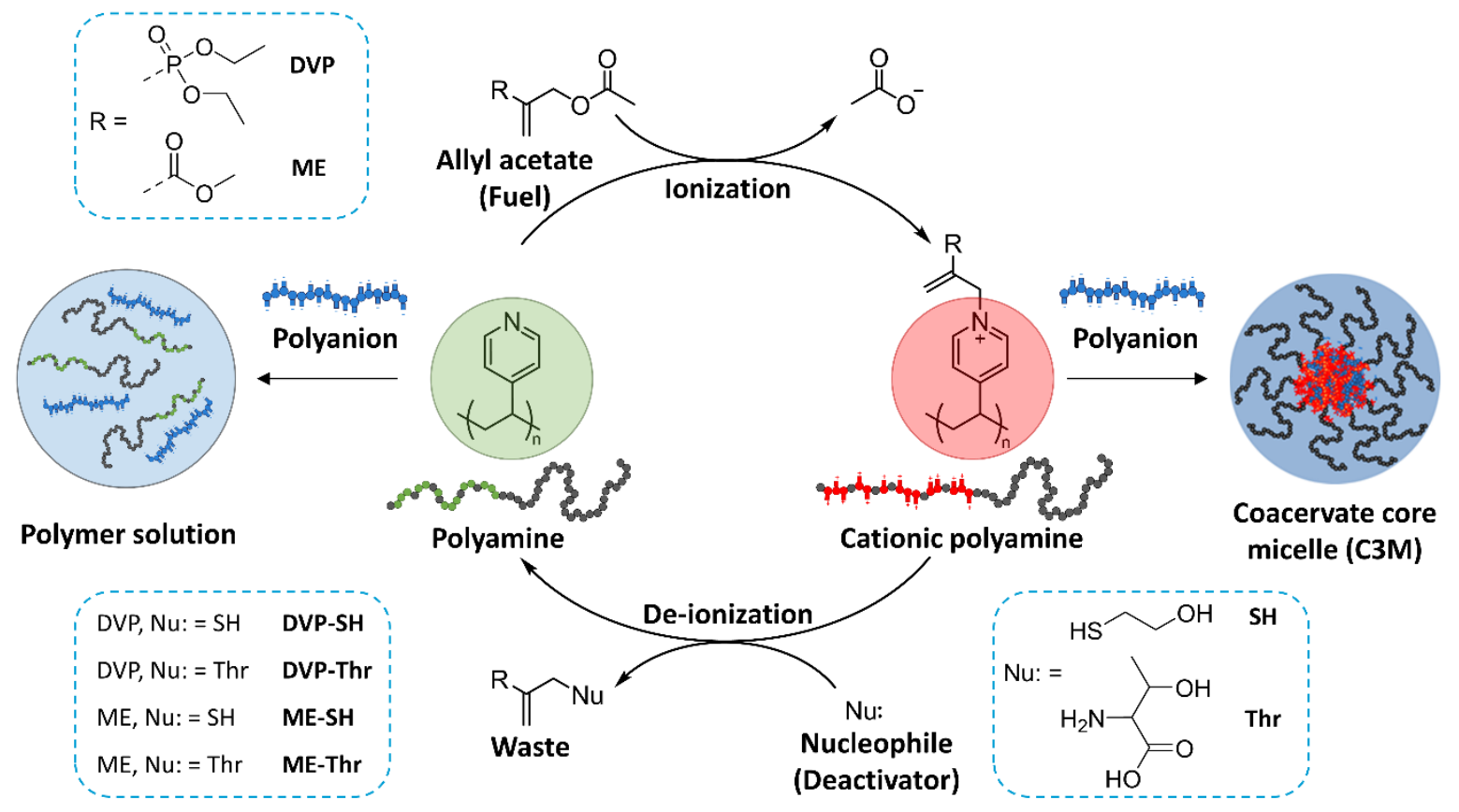

Scheme 1: Chemical reaction network $(\mathrm{CRN})$ for reversible ionization of polyamines leading to controlled coacervate core micelle $(\mathrm{C} 3 \mathrm{M})$ formation. Specifically, tertiary amine functionalised polymers undergo a nucleophilic substitution reaction with electron deficient allyl acetates (fuel), resulting in the formation of a cationic polyamine complex. A competing nucleophile (deactivator) can then participate a second substitution reaction with the polyamine complex, regenerating the starting tertiary amine polymer and producing a fuel-nucleophile waste product. When this cycle is conducted in the presence of suitable polyanions, polymer solution (unimer) to $\mathrm{C} 3 \mathrm{M}$ transitions are obtained. 


\section{Results and Discussion}

To realise reversible unimer to $\mathrm{C} 3 \mathrm{M}$ transitions induced by the allyl acetate $\mathrm{CRN}$, we first synthesised a polyamine block copolymer (P1, Figure 1a). This was prepared by a two-step reversible addition fragmentation chain-transfer (RAFT) process. ${ }^{37}$ First, dimethylacrylamide (DMA) was polymerised to form the water-soluble neutral block. This was then chain-extended by copolymerisation of 4-vinyl pyridine (4VP) and DMA to form the ionizable core block. A shift to lower retention times of a single GPC peak demonstrated successful chain extension, with the product P1 having a low dispersity $(\nexists=1.20)$ and molecular weights determined by GPC and conversion in excellent agreement $\left(M_{\mathrm{n}, \mathrm{GPC}}=32.9 \mathrm{kDa}, M_{\mathrm{n}, \mathrm{conv}}=32.8 \mathrm{kDa}\right) . \mathrm{We}$ selected pyridine as the tertiary amine functionality as it has a conjugate acid pKa of $5.2,{ }^{38}$ ensuring that negligible protonated (cationic) species exist at $\mathrm{pH} 7.4$ before addition of fuel. Water soluble DMA was incorporated into the ionizable core block to avert the formation of amphiphilic micelles in $\mathrm{pH} 7.4$ aqueous solutions, which was observed to occur for polyamine block copolymers containing only 4VP in the core.

To operate the reaction cycle we studied two different allyl acetate fuel molecules; diethyl $(\alpha-$ acetoxymethyl) vinylphosphonate (DVP) ${ }^{35}$ and methyl 2-(acetoxymethyl)acrylate (ME), ${ }^{36}$ the latter of which we found to be more reactive due to its stronger electron withdrawing $\mathrm{R}$ group. As an initial test we studied the allyl acetate ionization of P1 by combining DVP (1.0 eq.) with an aqueous solution of $\mathrm{P} 1$ (1.0 eq. 4VP, $20 \mathrm{mM}, \mathrm{pH} 7.4)$. This resulted in approximately $65 \%$ ionization of amine functionality after $120 \mathrm{~h}\left(\mathrm{P} 1_{\mathrm{D}}{ }^{23+}\right.$, Figure $\left.1 \mathbf{b}\right)$. Considering an average incorporation of $364 \mathrm{VP}$ units per polymer chain for $\mathrm{P} 1$, this equates to approximately 23 cationic units $\left(\mathrm{VP}_{\mathrm{D}}^{+}\right)$and 13 neutral (VP) per chain. Repeating the experiment with $\mathrm{ME}$ resulted in greater amine ionization $(80 \%)$ after only $5 \mathrm{~h}\left(\mathrm{P}_{\mathrm{M}^{29+}}\right.$, Figure 1c). Such conversion yielded an estimated 29 cationic pyridine units $\left(\mathrm{VP}_{\mathrm{M}}^{+}\right)$per chain. In both cases complete ionization was not observed, likely due to increasing charge repulsion effects as conversion 
increased. We next investigated whether $\mathrm{P} 1_{\mathrm{D}}{ }^{23+}$ could form $\mathrm{C} 3 \mathrm{Ms}$ when mixed with a polyanion, without which both $\mathrm{P} 1$ and $\mathrm{P} 1_{\mathrm{D}}{ }^{23+}$ are near indistinguishable polymer by DLS (Figure S11a). Considering the incomplete ionization, as well as the incorporation of neutral DMA in the core block, polyanion selection was likely to significantly influence C3M formation. This is because a minimum polyelectrolyte length and degree of ionization, together

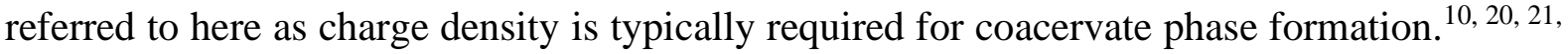
${ }^{39,40}$ Combining $\mathrm{P} 1_{\mathrm{D}}{ }^{23+}$ with various polyanions (Figure 1d) at equal concentration of cationic to anionic functionality ( $4 \mathrm{mM})$ yielded $\mathrm{C} 3 \mathrm{Ms}$ as demonstrated by increased number average diameter and light scatter by DLS (Figure S11c). Interestingly, TEM images demonstrated a morphological transition from a mixture of worm-like and spherical micelles to almost exclusively spherical micelles with increasing polyanion charge density (Figure 1e). A commercially obtained $200 \mathrm{kDa}$ poly(sodium 4-styrenesulfonate) (PSS) was selected for further study, as it formed relatively homogeneous spherical micelles with $\mathrm{P} 1_{\mathrm{D}}{ }^{23+}$.
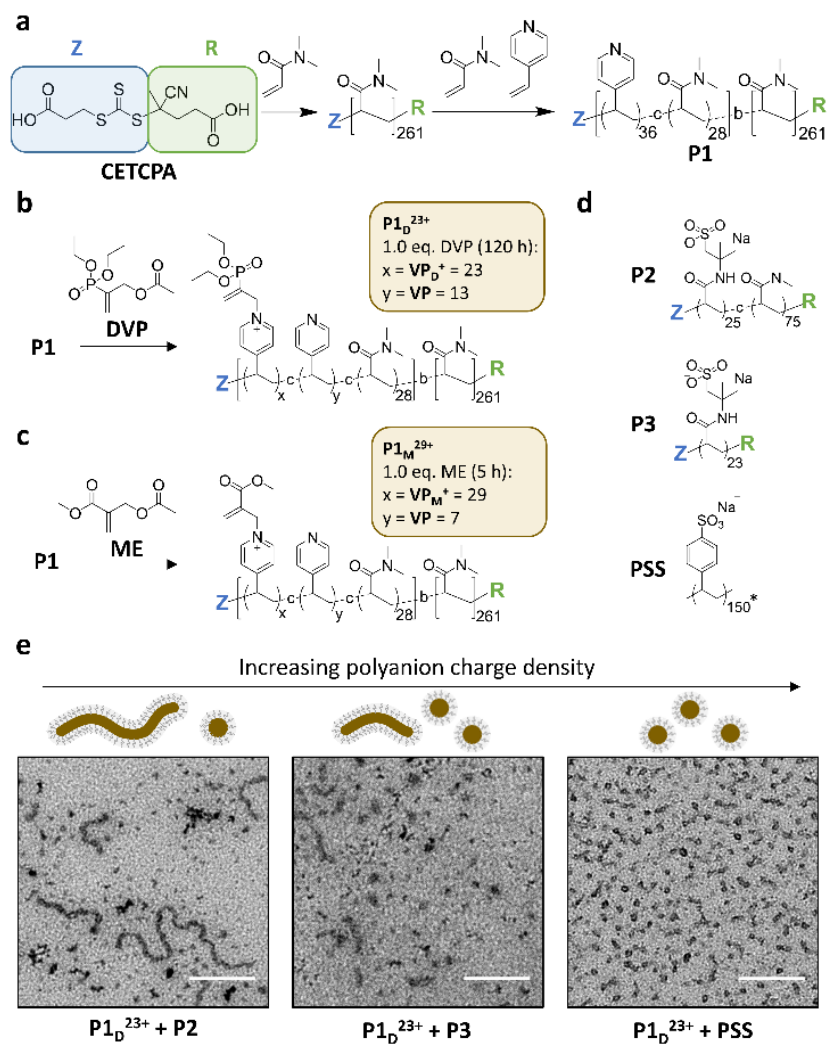
Figure 1. Polyamine block copolymer (P1) synthesis and assembly into C3Ms. a) Two step synthesis of P1 from RAFT polymerisation of DMA and 4VP with chain transfer agent (4((((2-carboxyethyl)thio)carbonothioyl)thio)-4-cyanopentanoic acid, CETCPA). b) Conversion of neutral VP units to cationic $\mathrm{VP}_{\mathrm{D}}{ }^{+}$in $\mathrm{P} 1(20 \mathrm{mM})$ by reaction with DVP (1.0 eq.) in $\mathrm{pH} 7.4$ PS buffer $(100 \mathrm{mM})$ yielding $\mathrm{P}_{\mathrm{D}}{ }^{23+}$. c) Conversion of neutral VP to cationic $\mathrm{VP}_{\mathrm{M}}{ }^{+}$in $\mathrm{P} 1(20$ $\mathrm{mM})$ by reaction with $\mathrm{ME}$ (1.0 eq.) in $\mathrm{pH} 7.4 \mathrm{PS}$ buffer $(100 \mathrm{mM})$ yielding $\mathrm{P} 1 \mathrm{M}^{29+}$. Acetate counterions in $\mathrm{P} 1 \mathrm{D}^{23+}$ and $\mathrm{P} 1 \mathrm{M}^{29+}$ omitted for visual clarity. d) Structures of polyanions combined $\mathrm{P} 1{ }_{\mathrm{D}}^{23+}$ during initial micelle formation studies. P2 and P3 were synthesised by RAFT polymerisation of 2-acrylamido-2-methylpropane sulfonic acid (AMPS) and DMA with CETCPA as the chain transfer agent. Poly(sodium 4-styrenesulfonate) (PSS) was commercially obtained with a reported MW of $200 \mathrm{kDa}$, while $M_{\mathrm{n}, \mathrm{GPC}}=31.5 \mathrm{kDa}$ was measured in our laboratory. *The average degree of polymerisation (150) as shown in the figure is therefore a conservative estimate based on $M_{\mathrm{n}, \mathrm{GPC}}$ to highlight its relatively high charge density.

e) TEM images and schematic illustration of coacervate core micelles (C3Ms) obtained from the combination of $\mathrm{P}_{\mathrm{D}^{23+}}$ with polyanions of varied charge density at $4 \mathrm{mM}$ amine and anionic functional group concentration (scale bar is $200 \mathrm{~nm}$ ). Samples were visualised with uranyl acetate staining. Additional TEM and DLS data are presented in Figures S11 - S12.

\section{Triggered micelle (de)formation}

Having established the formation of $\mathrm{C} 3 \mathrm{Ms}$ by combination of $\mathrm{P} 1_{\mathrm{D}}{ }^{23+}$ and PSS, we next sought to achieve allyl acetate triggered C3M formation in solution as depicted in Scheme 1. First, an aqueous buffered solution of P1 and PSS at equimolar amine and anionic functionality $(20 \mathrm{mM}$, $\mathrm{pH}$ 7.4) was prepared and transferred to a cuvette for in situ analysis of C3M formation by dynamic light scattering (DLS). To this mixture, we added DVP (1.0 eq. vs VP), resulting in an approximate 3 -fold increase in the light scatter intensity (scatter count) over $170 \mathrm{~h}$ (Figure 2a). In parallel, the experiment was performed without PSS and monitored by ${ }^{1} \mathrm{H}$ NMR to determine the extent of conversion from neutral VP to cationic $\mathrm{VP}_{\mathrm{D}}{ }^{+}$in $\mathrm{P} 1$ (Figure 2b). Note that PSS was excluded from the ${ }^{1} \mathrm{H}$ NMR experiment to avoid suppression of the charged polyamine complex protons due to inclusion in the micelle core. The scatter count increase and 
conversion to $\mathrm{VP}_{\mathrm{D}}{ }^{+}$were found to closely correlate, indicating the successful formation of micelles after in situ ionization of P1 with DVP. To complete the cycle in Scheme $\mathbf{1}$ and destroy the micelles, neutral VP needs to be regenerated by reaction of cationic $\mathrm{VP}_{\mathrm{D}}{ }^{+}$with a competing nucleophilic species. Preliminary experiments indicated that thiols readily perform this reaction and 2-mercaptoethanol ( $\mathrm{SH}, 1.0$ eq.) was added to both the DLS and ${ }^{1} \mathrm{H}$ NMR solutions at $170 \mathrm{~h}$. This promptly caused significant regeneration of $\mathrm{VP}\left(\mathrm{VP}_{\mathrm{D}}{ }^{+}\right.$conversion reduced from $64 \%$ to $7 \%$ within 40 minutes) along with formation of the DVP-SH waste product. This nucleophile triggered deionization of quaternized polyamine complexes in P1 resulted in rapid C3M deformation, as demonstrated by a reduction in DLS scatter count to near starting levels (from 11 to $5.5 \mathrm{Mcps}$ in 5 minutes). Additionally, since this process results in regeneration of the starting polyamine, it theoretically can be repeatedly cycled. Indeed, further addition of DVP (1.0 eq.) to the NMR experiment did demonstrate reformation of $\mathrm{VP}_{\mathrm{D}}{ }^{+}$ in P1 to similar conversion $(69 \%$, Figure S14a). However, no further DLS analysis was conducted since at the high polymer concentration (preferred for ${ }^{1} \mathrm{H}$ NMR analysis), DLS object diameter data was found to be noisy and unreliable, perhaps due to multiple scattering effects (Figure $S 14 c$ ).

To improve the quality of DLS data collected, we repeated the experiment at reduced polymer concentration ( $4 \mathrm{mM}$ ) and analysed it by DLS and TEM. This was conducted at both $25^{\circ} \mathrm{C}$ (like all other experiments) and $37.5^{\circ} \mathrm{C}$ to investigate if this cycle could operate under biologically relevant conditions. After addition of DVP to both samples, the scatter count increased significantly faster at $37.5^{\circ} \mathrm{C}$ than at $25^{\circ} \mathrm{C}$, with a respective doubling time of 12 and $56 \mathrm{~h}$ (Figure 2c). This demonstrated that mild heating can accelerate the reaction between DVP and the tertiary amine substrate, and as such the $37.5^{\circ} \mathrm{C}$ condition was selected for convenient study of two complete reaction cycles (full data from single cycle at $25^{\circ} \mathrm{C}$ in Figure S15). 
To achieve two cycles, sequential additions of DVP and SH were twice applied to a single solution of P1 and PSS (4 mM). Like the previous data collected at $20 \mathrm{mM}$, an increase in scatter count was observed after each addition of DVP, while each addition of SH gave a sharp decrease (Figure 2c). The DLS scatter count before and after addition of SH was found to be higher in the second cycle (from 10.0 to 4.6 Mcps) compared to the first (from 7.0 to 3.7 Mcps). This may be due to an incomplete first cycle reaction, waste accumulation or slight evaporation of aqueous solvent during the heated experiment (cuvette was parafilm covered). Importantly, an increase in the number average diameter from approximately 10 to $25 \mathrm{~nm}$ after addition of DVP was observed in both cycles, with thiol additions reverting the diameter back to the starting point (Figure 2d). Such a change in number average size indicates a transition from polymer solution $(10 \mathrm{~nm})$ to spherical micelles $(25 \mathrm{~nm})$, an explanation further supported by TEM images taken at the end of each addition. Here, corresponding transitions from faint wormlike species to darker spherical objects of $23.7 \pm 3.6 \mathrm{~nm}$ and $14.0 \pm 3.4 \mathrm{~nm}$ were observed following the first and second DVP addition, respectively (Figure 2e). Combined these data demonstrate triggered formation and deformation of C3Ms after addition of DVP and $\mathrm{SH}$, respectively. 

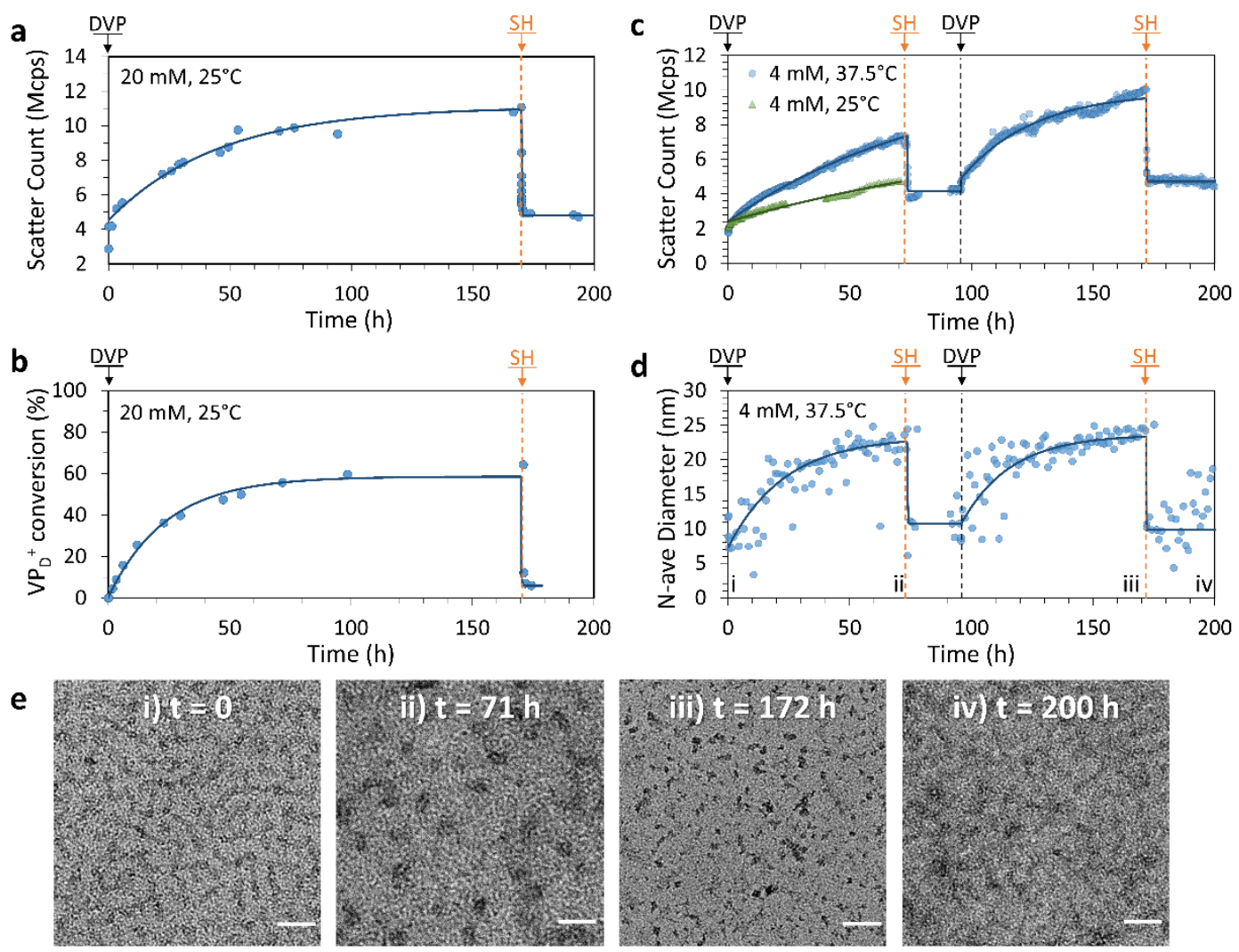

Figure 2. Signal induced C3M (de)formation from sequential 1.0 eq. additions of DVP and SH to a mixture of P1 and PSS in $100 \mathrm{mM}$ PS pH 7.4 buffer. Experiment was first conducted at $25^{\circ} \mathrm{C}, 20 \mathrm{mM}$ P1 and PSS with a) change in scatter count observed by DLS and b) matching conversion of VP to $\mathrm{VP}_{\mathrm{D}}{ }^{+}$in $\mathrm{P} 1$ monitored by ${ }^{1} \mathrm{H}$ NMR (without PSS to avoid suppression of micelle core signals). The experiment was repeated at $37.5^{\circ} \mathrm{C}, 4 \mathrm{mM}$ and monitored by DLS and TEM. c) Change in scatter count (70 $\mathrm{h}$ after first DVP addition is also shown for $25^{\circ} \mathrm{C}$ ) and d) number average diameter over time, time points corresponding to TEM images marked with Roman numerals. e) Representative TEM images taken throughout $37.5^{\circ} \mathrm{C}, 4 \mathrm{mM}$ cycle at: i) $\mathrm{t}=0$ (polymer solution), ii) $\mathrm{t}=71 \mathrm{~h}(\mathrm{C} 3 \mathrm{Ms})$, iii) $\mathrm{t}=172 \mathrm{~h}(\mathrm{C} 3 \mathrm{Ms})$ and iv) $200 \mathrm{~h}$ (polymer solution) with scale bars at $50 \mathrm{~nm}$. Exponential curves drawn after each addition are to guide the eye. Additional DLS, ${ }^{1} \mathrm{H}$ NMR and TEM data including full details of $4 \mathrm{mM}, 25^{\circ} \mathrm{C}$ experiment can be found in the Figures S13-S17.

With this promising result we decided to investigate if ME, a more reactive allyl acetate could be utilised to achieve the same $\mathrm{C} 3 \mathrm{M}$ switching behaviour in an accelerated fashion. In this case, addition of ME (1.0 eq. vs VP) to a $20 \mathrm{mM}$ aqueous solution of P1 and PSS resulted in a similar 
3-fold increase in DLS scatter count over only $2 \mathrm{~h}$. This was in agreement with ${ }^{1} \mathrm{H}$ NMR measurements (without PSS), which indicated approximately $60 \%$ conversion of $\mathrm{VP}$ to $\mathrm{VP}_{\mathrm{M}}{ }^{+}$ after $2 \mathrm{~h}$ and a peak conversion of $80 \%$ after $5 \mathrm{~h}$ (Figure 3a - b). Interestingly the scatter count remained constant after $1.5 \mathrm{~h}$, which indicates a critical conversion near $60 \%$, beyond which little change in the extent of micelle formation is observed. Also of note, is the slight decrease in NMR conversion from 5 to $40 \mathrm{~h}(80 \%$ to $70 \%)$, which we attribute to phosphate nucleophilic substitution on the $\mathrm{VP}_{\mathrm{M}}{ }^{+}$quaternary complex (Figure S18). Addition of $\mathrm{SH}$ (1.0 eq.) then rapidly (within 10 minutes) regenerated neutral $\mathrm{VP}$ (conversion to $\mathrm{VP}_{\mathrm{M}}{ }^{+}$reduced from $70 \%$ to 20\%) and yielded the ME-SH waste product. Like the DVP based system, this triggered reduction in ionization of $\mathrm{P} 1$ appears to result in $\mathrm{C} 3 \mathrm{M}$ destruction as evidenced by a reduction in scatter count from 11.3 to 4.6 Mcps. Re-formation of $\mathrm{VP}_{\mathrm{M}}^{+}$in a second cycle was found to proceed to a similar conversion (78\%) $4 \mathrm{~h}$ after a further addition of ME (1.0 eq., Figure S21a), indicating ability for use in multiple cycles. NMR experiments also identified the formation of a double Michael adduct (ME-2SH, two additions of SH to ME), which formed in appreciable quantity when an excess of SH was added (Figures S18-S19).

We then conducted a complete two cycle experiment analysed by DLS at $4 \mathrm{mM}$ polymer concentration. As expected, each addition of ME (1.0 eq.) led to an increase in scatter count over $7 \mathrm{~h}$ (slower rate than at $20 \mathrm{mM}$ due to dilution), while each addition of SH (1.0 eq.) gave a sharp decrease (Figure 3c). Promisingly, the peak and minimum scatter counts after ME and SH addition were found to be similar for each cycle (within 15\%), indicating excellent repeatability of the reaction cycle for $\mathrm{C} 3 \mathrm{M}$ formation. A shift in number average diameter from approximately 10 to $25 \mathrm{~nm}$ after addition of ME was observed, which reverted to approximately $10 \mathrm{~nm}$ after addition of SH (Figure 3d), matching the endpoints observed with DVP. TEM images taken at the end of each addition showed transitions from faint wormlike species to darker spherical objects of $23.3 \pm 4.4 \mathrm{~nm}$ and $17.8 \pm 2.6 \mathrm{~nm}$ after the first and second $\mathrm{ME}$ 
addition, respectively (Figure 3e). Together, these results demonstrate ME triggered formation of C3Ms in a similar but accelerated manner to that observed with DVP.
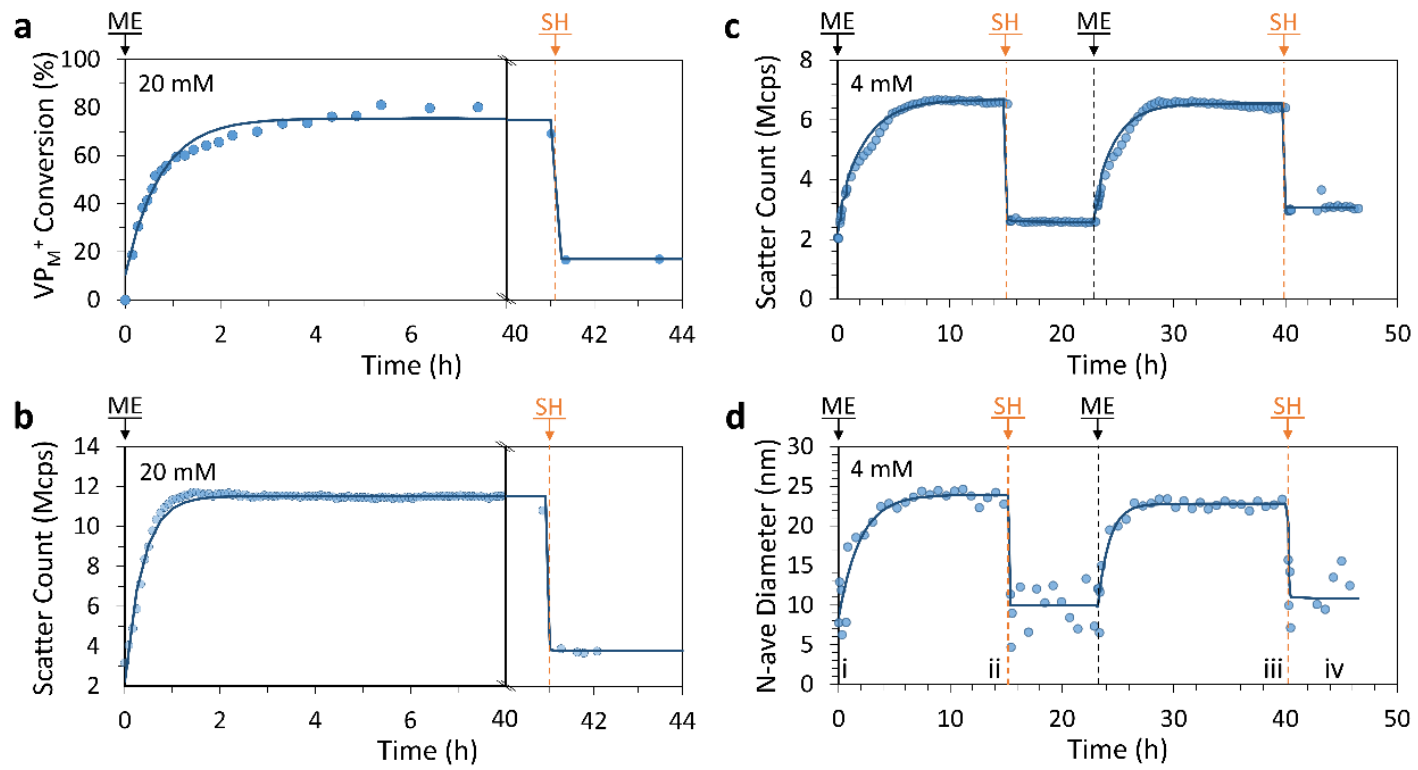

$\mathbf{e}$
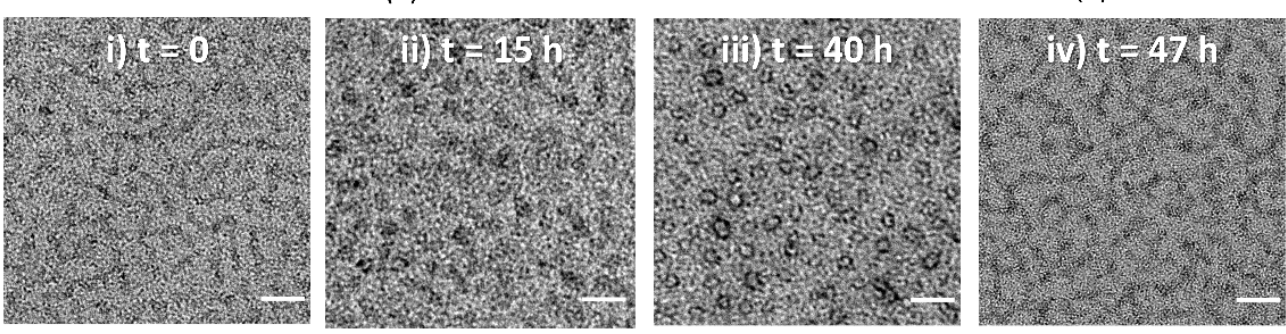

Figure 3. Signal induced C3M (de)formation from sequential 1.0 eq. additions of ME and SH to a mixture of P1 and PSS in $100 \mathrm{mM}$ PS pH 7.4 buffer at $25^{\circ} \mathrm{C}$. Experiment was first conducted at $20 \mathrm{mM} \mathrm{P} 1$ and PSS with a) conversion of VP to $\mathrm{VP}_{\mathrm{M}}{ }^{+}$in $\mathrm{P} 1$ monitored by ${ }^{1} \mathrm{H}$ NMR (without PSS to avoid suppression of micelle core signals) and b) matching DLS scatter count data. The experiment was repeated at $4 \mathrm{mM}$ and monitored by DLS and TEM. c) Change in scatter count and d) number average diameter over time (time points corresponding to TEM images marked with Roman numerals). e) Representative TEM images taken throughout $4 \mathrm{mM}$ cycle at: i) $\mathrm{t}=0$ (polymer solution), ii) $\mathrm{t}=15 \mathrm{~h}(\mathrm{C} 3 \mathrm{Ms})$, iii) $\mathrm{t}=40 \mathrm{~h}(\mathrm{C} 3 \mathrm{Ms})$ and iv) $47 \mathrm{~h}$ (polymer solution). Scale bars $50 \mathrm{~nm}$. Exponential curves drawn after each addition are to guide the eye. Additional DLS, ${ }^{1} \mathrm{H}$ NMR and TEM data can be found in the Figures S18-S24. 


\section{Transient micelle deformation (excess fuel)}

So far, we have established the ability to trigger transitions from unimers to C3Ms by sequential addition of allyl acetates and thiols to an aqueous solution of P1 and PSS. These transitions are essentially triggered switching between equilibrium states. To access non-equilibrium states, we investigated the use of a molar excess of allyl acetates, thereby allowing kinetics to control the assembly and disassembly processes and the composition of the mixture at any given time. To this end, 3.0 equivalents of DVP were added to a solution of P1 and PSS (both $4 \mathrm{mM}$ ). As expected, we observed a slow but strong increase in the DLS scatter count and number average size, with the number average size plateauing after $100 \mathrm{~h}$ (increase from approximately 10 to $22 \mathrm{~nm}$ ). Over the same time period $25 \%$ of the available DVP was consumed, indicating formation of $\mathrm{VP}_{\mathrm{D}}^{+}$in $\mathrm{P} 1$ (up to approx. $82 \%$, Figure $S 26 a$ ). At this point the system still contained approx. 2.3 eq. of unreacted DVP as well as the newly formed C3Ms. At $119 \mathrm{~h} \mathrm{SH}$ (1.0 eq.) was added, which rapidly (within $2 \mathrm{~h}$ ) and nearly exclusively reacted with the cationic $\mathrm{VP}_{\mathrm{D}}{ }^{+}$to produce neutral VP in P1 and the waste product DVP-SH (Figure 4a-c). This can be concluded as only $3 \%$ of unreacted DVP was consumed during the formation of $23 \%$ DVPSH. Similarly, $2 \mathrm{~h}$ after $\mathrm{SH}$ addition, both the scatter count and number average size had reduced to near starting levels (from 6.0 Mcps and $21 \mathrm{~nm}$ to $3.0 \mathrm{Mcps}$ and $10 \mathrm{~nm}$ ), confirming the expected C3M disassembly. Importantly, since there is still DVP in the system, this state of disassembly is not at equilibrium and spontaneous $\mathrm{C} 3 \mathrm{M}$ reformation is expected after further generation of cationic $\mathrm{VP}_{\mathrm{D}}{ }^{+}$species in $\mathrm{P} 1$. Indeed, after $312 \mathrm{~h}$ the number average size had returned to around $20 \mathrm{~nm}$ and the scatter count had reached $80 \%$ of the previous cycle peak. At this point DVP conversion had reached $50 \%$, indicating a similar $\mathrm{VP}_{\mathrm{D}}{ }^{+}$species generation as in the first cycle. It could therefore be concluded that the system had returned to a near equilibrium C3M state. In an attempt to attain a second transient disassembly, a second SH (1.0 eq.) addition was made at $312 \mathrm{~h}$. This resulted in further formation of DVP-SH (up to $53 \%$ ) as 
well as reduced scatter count and number average size (from 4.8 Mcps and $20 \mathrm{~nm}$ to $2.8 \mathrm{Mcps}$ and $10 \mathrm{~nm}$ ). Reformation of C3Ms after this second disassembled state, was however, not observed despite the system theoretically containing enough DVP to allow for further reformation. Discrete DLS measurements recorded up to $t=1000 \mathrm{~h}$ indicated only a slight increase in scatter count (3.3 Mcps).

After successfully attaining a non-equilibrium disassembled state with DVP as the fuel we investigated if similar behaviour could be accessed with ME. Similarly, C3Ms were initially formed by addition of ME (3.0 eq.) to a solution of P1 and PSS (both $4 \mathrm{mM}$ ). The higher reactivity of $\mathrm{ME}$ resulted in rapid increases in scatter count and number average size, which plateaued after only $1 \mathrm{~h}$, indicating stable formation of micelles. At $18 \mathrm{~h} 30 \%$ of ME was consumed and $\mathrm{SH}$ (1.0 eq.) was added, resulting in a short-lived non-equilibrium $\mathrm{C} 3 \mathrm{M}$ disassembly. This was demonstrated by reductions in both scatter count and number average size, with a minimum in these parameters 10 minutes after $\mathrm{SH}$ addition (from $6.0 \mathrm{Mcps}$ and 21 $\mathrm{nm}$ to $3.7 \mathrm{Mcps}$ and $13 \mathrm{~nm}$ ). This was then followed by spontaneous C3M reformation and within $2 \mathrm{~h}$ both parameters had returned to near starting values. Interestingly, unlike the DVP based experiment a second transient disassembled state was attained after a second addition of $\mathrm{SH}$ (1.0 eq.) at $42 \mathrm{~h}$. The time for C3M reformation was longer in this case (16 h), likely related to the reduced excess of remaining ME. TEM images from a sample taken at $65 \mathrm{~h}$ confirmed the reformation of spherical micelle structures after the second $\mathrm{SH}$ addition of $17.5 \pm 2.7 \mathrm{~nm}$ (Figure S29b). Lastly, a final SH (1.0 eq.) addition resulted in sustained C3M deformation (scatter count remained at 3.2 Mcps), with complete conversion of the ME to its waste products (ME-SH and ME-2SH) observed by ${ }^{1} \mathrm{H}$ NMR (Figure 4d-f). It is of note that despite the higher reactivity of ME, little direct reaction was observed between ME and $\mathrm{SH}$. 
Overall, these experiments demonstrate how pairing the relatively rapid kinetics of $\mathrm{SH}$ triggered P1 deionization with a slower, but in stoichiometric excess ionization of P1 by an allyl acetate, allows access to a transient non-equilibrium disassembly.
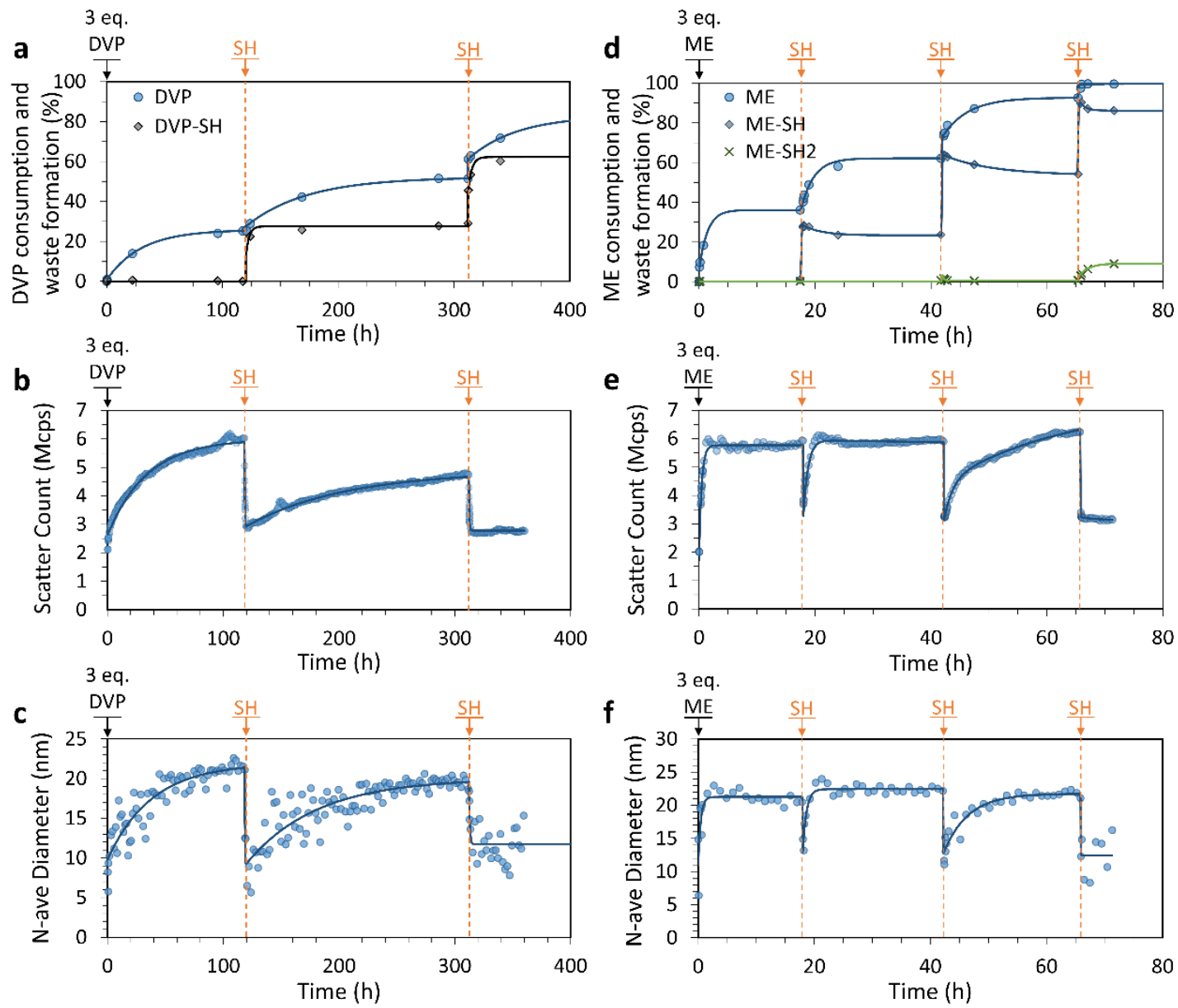

Figure 4. Transient $\mathrm{C} 3 \mathrm{M}$ disassembly triggered by sequential 1.0 eq. additions of $\mathrm{SH}$ to a mixture of P1 and PSS (4 mM), where excess (3.0 eq.) DVP (a-c) or ME (d-f) was added at t $=0$ to pre-form the micelles. All experiments were conducted in $100 \mathrm{mM}$ PS pH 7.4 buffer. The process was monitored by ${ }^{1} \mathrm{H}$ NMR (a, d), where only allyl acetate consumption and waste formation was quantified since PSS was included in the ${ }^{1} \mathrm{H}$ NMR experiment, masking the micelle core signals. C3M formation was confirmed by DLS measurements quantitating the scatter count (b, e) and number average diameter (c, f). Exponential curves drawn after each addition are to guide the eye. Additional DLS, ${ }^{1} \mathrm{H}$ NMR and TEM data can be found in Figures S25-S29. 


\section{Dissipative micelle formation}

We next envisaged that non-equilibrium assembled structures could be attained by reducing the reactivity of the deactivating nucleophile species such that the ionization reaction is faster than the de-ionization. In this way, a system could be constructed with an allyl acetate acting as a chemical fuel, allowing for an initial accumulation of the ionized VP units causing C3M formation. If the nucleophile is present in excess it should eventually consume all ionized VP species after the fuel is depleted, ultimately leading to $\mathrm{C} 3 \mathrm{M}$ disassembly. To achieve this behaviour, threonine (Thr), a relatively weak, primary amine nucleophile was selected based on our earlier work. ${ }^{35}$ After trialling various concentrations of fuel, Thr, P1 and PSS, the following were found to be most optimal. First, we prepared $\mathrm{pH} 7.4$ buffered solutions containing P1 (4 mM), PSS (4 mM) and Thr $(32 \mathrm{mM})$ in triplicate. After $10 \mathrm{~h}$ to record baseline DLS data $(2.2 \pm 0.16 \mathrm{Mcps}, 9.6 \pm 4.1 \mathrm{~nm})$, DVP fuel $(2.0$ e.q, $8 \mathrm{mM})$ was added to trigger C3M formation, leading to an increase in scatter count and number average size (Figure 5a - b). These values peaked at $70-90 \mathrm{~h}(4.0 \pm 0.21 \mathrm{Mcps}, 17 \pm 3.3 \mathrm{~nm})$, after which a steady decrease in both parameters was observed. Between 420 and $500 \mathrm{~h}$ the scatter count and number average size had returned to near starting levels $(2.5 \pm 0.22 \mathrm{Mcps}, 12 \pm 4.4 \mathrm{~nm})$, indicating transient formation of C3Ms in solution. Note that scatter count and number average size values reported are an average \pm standard deviation across the triplicate measurements for time period specified (where they are assumed to be $\sim$ constant).

In a similar case, ME fuel (2.0 e.q, $8 \mathrm{mM}$ ) was added to $\mathrm{pH} 7.4$ buffered solutions containing P1 (4 mM), PSS (4 mM) and Thr (20 mM). In this case, we recorded baseline DLS data for the first $2 \mathrm{~h}(2.3 \pm 0.16 \mathrm{Mcps}, 9.4 \pm 4.1 \mathrm{~nm})$, after which fuel addition caused rapid C3M formation (Figure 5d - e). Scatter count $(4.3 \pm 0.15 \mathrm{Mcps})$ and number average size $(18 \pm 1.6 \mathrm{~nm})$ peak values were recorded between 3 and $4 \mathrm{~h}(1-2 \mathrm{~h}$ after ME addition). A spontaneous C3M deformation followed, with scatter count reduced to $2.9 \pm 0.1 \mathrm{Mcps}$ at $16 \mathrm{~h}$ where it remained 
for the rest of the experiment. The number average size underwent a slower reduction to reach $11 \pm 4.7 \mathrm{~nm}$ between 24 and $30 \mathrm{~h}$.

It is of note that for both DVP and ME fuelled experiments, the peak number average size and scatter count were lower than those attained for signal induced $\mathrm{C} 3 \mathrm{M}$ formation. This suggests a reduced or incomplete association of the polymers into micelles. Indeed, ${ }^{1} \mathrm{H}$ NMR measurements indicated peak conversion to ionized pyridine species of only $27 \%\left(\mathrm{VP}_{\mathrm{D}}{ }^{+}\right.$, Figure 5c) and $34 \%\left(\mathrm{VP}_{\mathrm{M}}^{+}\right.$, Figure 5f). This estimation was obtained by quantitating the excess consumption of fuel (DVP or ME) compared to waste(s) production. TEM images from samples taken at peak aggregation (100 $\mathrm{h}$ for DVP and $3.5 \mathrm{~h}$ for ME) were able to identify spherical micelle objects of $12.8 \pm 4.7 \mathrm{~nm}$ and $16.2 \pm 3.4 \mathrm{~nm}$, respectively. These are slightly smaller than the diameters from corresponding signal induced experiments and, unlike all previous measurements these were only able to be visualised effectively without uranyl acetate stain (Figures $S 36-S 37$ ). With these considerations in mind, the results pleasingly demonstrate transient (partial) association of polymers into C3Ms with fuel dependent lifetimes. 

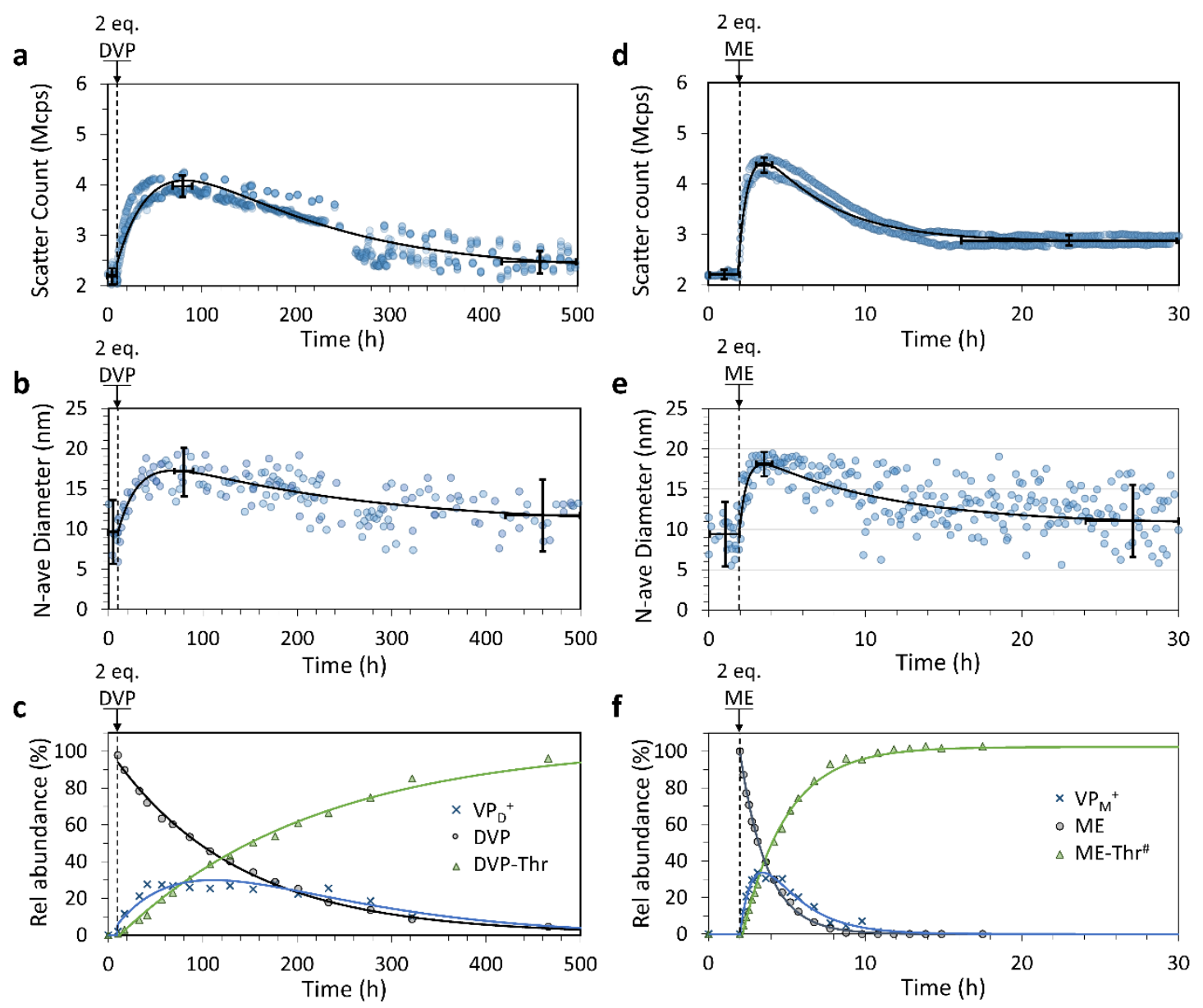

Figure 5. Fuel-driven transient $\mathrm{C} 3 \mathrm{M}$ assembly by addition of 2.0 eq. fuel (DVP or ME) to a solution of P1 (4 mM), PSS (4 mM) and Thr (32 mM for a-c and $20 \mathrm{mM}$ for d-f). Solutions were maintained at $\mathrm{pH} 7.4$ by $250 \mathrm{mM}$ PS buffer. DLS measurements run in triplicate on independent samples quantitating the scatter count (a,d) and number average diameter (b, e). Two-exponential curves (black line) were fit to the data. Average values (centre of cross) and standard deviation (vertical bars) before fuel addition, at peak micelle formation and at the end were analysed assuming constant values over the time span shown by the horizontal bars. The process was also monitored by ${ }^{1} \mathrm{H}$ NMR (c, f), where only fuel and waste signals were quantitated since PSS was included in the ${ }^{1} \mathrm{H}$ NMR experiment. In the case of the ME fuelled experiment multiple waste products were formed, the total of which is described by ME-Thr\#. Values for $\mathrm{VP}_{\mathrm{D}}{ }^{+}$and $\mathrm{VP}_{\mathrm{M}}{ }^{+}$are only an estimate, obtained by subtracting the amount of waste produced from the amount of fuel consumed. The values are expressed as a \% conversion of VP in P1. Single and two-exponential curves are drawn to guide the eye. The break in DLS data in figures $\mathrm{a}$ and $\mathrm{b}$ between 240 and $280 \mathrm{~h}$ are due to a required filtration of all samples to remove an unidentified biological growth that began to accumulate in each sample. Filtration was through a $0.45 \mu \mathrm{m}$ filter and therefore should not significantly remove the $\sim 20 \mathrm{~nm}$ micelles, 
for further discussion see Figures $S 32$ - S33. Additional DLS, ${ }^{1} \mathrm{H}$ NMR and TEM data can be found in Figures S30-S36.

\section{Conclusions}

In conclusion, we have demonstrated the formation of complex coacervate core micelles (C3Ms) containing polycation blocks generated from the quaternization of a neutral tertiary polyamine by electron deficient allyl acetates. The C3Ms can then be disassembled by addition of a competing nucleophile, regenerating the starting neutral tertiary amine segments in the polyamine. This process essentially recovers the starting polyamine, allowing for repeated cycling of $\mathrm{C} 3 \mathrm{M}$ (de)formation by coacervate phase modulation at constant $\mathrm{pH}$. Two allyl acetates were investigated, with the rate of $\mathrm{C} 3 \mathrm{M}$ formation found to be highly dependent on allyl acetate reactivity, giving timescales of formation ranging from hours to days. Signal induced $\mathrm{C} 3 \mathrm{M}$ formation and deformation was repeated for two complete cycles by sequential additions of an allyl acetate and 2-mercaptoethanol. In the initial presence of excess allyl acetate, additions of 2-mercaptoethanol after micelle formation resulted in transient deformation of C3Ms. Lastly, by optimising the kinetics of micelle formation and deformation, we demonstrated allyl acetate fuel-driven C3M formation using a starting excess of a weak nucleophile (threonine). We anticipate this nucleophile controlled transient coacervate phase formation will inspire developments into triggered nucleic acid delivery and non-equilibrium protocell behaviour. 


\section{Acknowledgments}

The authors acknowledge financial support by the European Research Council (ERC Consolidator Grant 726381). We also thank A. Aerts (TU Eindhoven) for valuable assistance in preliminary GPC polymer characterisation.

\section{References}

1. Urban, M. W., Introduction to responsive materials for healthcare diagnostics. J. Mater. Chem. B 2020, 8 (29), 6168-6169.

2. Nakashima, K. K.; van Haren, M. H. I.; André, A. A. M.; Robu, I.; Spruijt, E., Active coacervate droplets are protocells that grow and resist Ostwald ripening. Nat. Commun. 2021, $12(1), 3819$.

3. Li, P.; Xia, Y.; Hao, J.; Wang, X., Transient Healability of Metallosupramolecular Polymer Networks Mediated by Kinetic Control of Competing Chemical Reactions. Macromolecules 2020, 53 (8), 2856-2863.

4. Cabral, H.; Miyata, K.; Osada, K.; Kataoka, K., Block Copolymer Micelles in Nanomedicine Applications. Chem. Rev. 2018, 118 (14), 6844-6892.

5. Singh, N.; Formon, G. J.; De Piccoli, S.; Hermans, T. M., Devising Synthetic Reaction Cycles for Dissipative Nonequilibrium Self - Assembly. Adv. Mater. 2020, 32 (20), 1906834.

6. Rapoport, N., Physical stimuli-responsive polymeric micelles for anti-cancer drug delivery. Prog. Polym. Sci. 2007, 32 (8-9), 962-990.

7. De, S.; Klajn, R., Dissipative self - assembly driven by the consumption of chemical fuels. Adv. Mater. 2018, 30 (41), 1706750.

8. Te Brinke, E.; Groen, J.; Herrmann, A.; Heus, H. A.; Rivas, G.; Spruijt, E.; Huck, W. T., Dissipative adaptation in driven self-assembly leading to self-dividing fibrils. Nat. Nanotechnol. 2018, 13 (9), 849-855.

9. $\quad$ van Rossum, S. A.; Tena-Solsona, M.; van Esch, J. H.; Eelkema, R.; Boekhoven, J., Dissipative out-of-equilibrium assembly of man-made supramolecular materials. Chem. Soc. Rev. 2017, 46 (18), 5519-5535.

10. van der Gucht, J.; Spruijt, E.; Lemmers, M.; Cohen Stuart, M. A., Polyelectrolyte complexes: bulk phases and colloidal systems. J. Colloid Interface Sci. 2011, 361 (2), 407-22. 11. Sing, C. E.; Perry, S. L., Recent progress in the science of complex coacervation. Soft Matter 2020, 16 (12), 2885-2914.

12. Horn, J. M.; Kapelner, R. A.; Obermeyer, A. C., Macro- and Microphase Separated Protein-Polyelectrolyte Complexes: Design Parameters and Current Progress. Polymers 2019, 11 (4).

13. Blocher, W. C.; Perry, S. L., Complex coacervate-based materials for biomedicine. Wiley Interdiscip Rev Nanomed Nanobiotechnol 2017, 9 (4).

14. Harada, A.; Kataoka, K., Formation of Polyion Complex Micelles in an Aqueous Milieu from a Pair of Oppositely-Charged Block-Copolymers with Poly(Ethylene Glycol) Segments. Macromolecules 1995, 28 (15), 5294-5299.

15. Voets, I. K.; de Keizer, A.; Cohen Stuart, M. A., Complex coacervate core micelles. Adv. Colloid Interface Sci. 2009, 147-148, 300-18. 
16. Voets, I. K., Electrostatically Driven Assembly of Polyelectrolytes. In Fluorescence Studies of Polymer Containing Systems, Procházka, K., Ed. Springer International Publishing: Cham, 2016; pp 65-89.

17. Mills, C. E.; Obermeyer, A.; Dong, X.; Walker, J.; Olsen, B. D., Complex Coacervate Core Micelles for the Dispersion and Stabilization of Organophosphate Hydrolase in Organic Solvents. Langmuir 2016, 32 (50), 13367-13376.

18. Shi, B.; Zheng, M.; Tao, W.; Chung, R.; Jin, D.; Ghaffari, D.; Farokhzad, O. C., Challenges in DNA Delivery and Recent Advances in Multifunctional Polymeric DNA Delivery Systems. Biomacromolecules 2017, 18 (8), 2231-2246.

19. Uchida, S.; Kataoka, K., Design concepts of polyplex micelles for in vivo therapeutic delivery of plasmid DNA and messenger RNA. J Biomed Mater Res A 2019, 107 (5), 978-990. 20. Rumyantsev, A. M.; Zhulina, E. B.; Borisov, O. V., Scaling Theory of Complex Coacervate Core Micelles. ACS Macro Lett. 2018, 7 (7), 811-816.

21. Chollakup, R.; Beck, J. B.; Dirnberger, K.; Tirrell, M.; Eisenbach, C. D., Polyelectrolyte Molecular Weight and Salt Effects on the Phase Behavior and Coacervation of Aqueous Solutions of Poly(acrylic acid) Sodium Salt and Poly(allylamine) Hydrochloride. Macromolecules 2013, 46 (6), 2376-2390.

22. Lemmers, M.; Sprakel, J.; Voets, I. K.; van der Gucht, J.; Cohen Stuart, M. A., Multiresponsive reversible gels based on charge-driven assembly. Angew. Chem. Int. Ed. Engl. 2010, 49 (4), 708-11.

23. Tsitsilianis, C., Self-assembling Hydrogels from $\mathrm{pH}$-Responsive Ionic Block Copolymers. In Hydrogels: Recent Advances, Thakur, V. K.; Thakur, M. K., Eds. Springer Singapore: Singapore, 2018; pp 259-295.

24. Miyata, K.; Oba, M.; Nakanishi, M.; Fukushima, S.; Yamasaki, Y.; Koyama, H.; Nishiyama, N.; Kataoka, K., Polyplexes from Poly(aspartamide) Bearing 1,2-Diaminoethane Side Chains Induce pH-Selective, Endosomal Membrane Destabilization with Amplified Transfection and Negligible Cytotoxicity. J. Am. Chem. Soc. 2008, 130 (48), 16287-16294.

25. Harada, A.; Kataoka, K., On-Off Control of Enzymatic Activity Synchronizing with Reversible Formation of Supramolecular Assembly from Enzyme and Charged Block Copolymers. J. Am. Chem. Soc. 1999, 121 (39), 9241-9242.

26. van Hees, I. A.; Swinkels, P. J. M.; Fokkink, R. G.; Velders, A. H.; Voets, I. K.; van der Gucht, J.; Kamperman, M., Self-assembly of oppositely charged polyelectrolyte block copolymers containing short thermoresponsive blocks. Polym. Chem. 2019, 10 (23), 31273134.

27. Cingil, H. E.; Meertens, N. C. H.; Voets, I. K., Temporally Programmed Disassembly and Reassembly of C3Ms. Small 2018, 14 (46), e1802089.

28. Facciotti, C.; Saggiomo, V.; Bunschoten, A.; ten Hove, J. B.; Rood, M. T. M.; van Leeuwen, F. W. B.; Velders, A. H., Assembly, Disassembly and Reassembly of Complex Coacervate Core Micelles with Redox-Responsive Supramolecular Cross-Linkers. ChemSystemsChem 2020, 2 (4), e1900032.

29. Geng, W.-C.; Liu, Y.-C.; Zheng, Z.; Ding, D.; Guo, D.-S., Direct visualization and real-time monitoring of dissipative self-assembly by synchronously coupled aggregationinduced emission. Mater. Chem. Front. 2017, 1 (12), 2651-2655.

30. Hoon Jeong, J.; Christensen, L. V.; Yockman, J. W.; Zhong, Z.; Engbersen, J. F. J.; Jong Kim, W.; Feijen, J.; Wan Kim, S., Reducible poly(amido ethylenimine) directed to enhance RNA interference. Biomaterials 2007, 28 (10), 1912-1917.

31. Oupicky, D.; Parker, A. L.; Seymour, L. W., Laterally stabilized complexes of DNA with linear reducible polycations: strategy for triggered intracellular activation of DNA delivery vectors. J. Am. Chem. Soc. 2002, 124 (1), 8-9. 
32. Semenov, S. N.; Wong, A. S. Y.; van der Made, R. M.; Postma, S. G. J.; Groen, J.; van Roekel, H. W. H.; de Greef, T. F. A.; Huck, W. T. S., Rational design of functional and tunable oscillating enzymatic networks. Nat. Chem 2015, 7 (2), 160-165.

33. Aumiller, W. M.; Keating, C. D., Phosphorylation-mediated RNA/peptide complex coacervation as a model for intracellular liquid organelles. Nat. Chem 2016, 8 (2), 129-137.

34. Donau, C.; Späth, F.; Sosson, M.; Kriebisch, B. A. K.; Schnitter, F.; Tena-Solsona, M.; Kang, H.-S.; Salibi, E.; Sattler, M.; Mutschler, H.; Boekhoven, J., Active coacervate droplets as a model for membraneless organelles and protocells. Nat. Commun. 2020, 11 (1), 5167.

35. Klemm, B.; Lewis, R.; Piergentili, I.; Eelkema, R., Temporally programmed polymer - solvent interactions using a chemical reaction network. ChemRxiv. Cambridge: Cambridge Open Engage; 2021; This content is a preprint and has not been peer-reviewed. 2021. doi: 10.33774/chemrxiv-2021-jh5jm

36. Zhuang, J.; Zhao, B.; Meng, X.; Schiffman, J. D.; Perry, S. L.; Vachet, R. W.; Thayumanavan, S., A programmable chemical switch based on triggerable Michael acceptors. Chem. Sci. 2020, 11 (8), 2103-2111.

37. Moad, G.; Rizzardo, E.; Thang, S. H., Living Radical Polymerization by the RAFT Process - A Third Update. Aust. J. Chem. 2012, 65 (8), 985-1076.

38. Dewick, P. M., Essentials of Organic Chemistry: For Students of Pharmacy, Medicinal Chemistry and Biological Chemistry. John Wiley \& Sons, Ltd: West Sussex, England, 2006.

39. van der Burgh, S.; de Keizer, A.; Cohen Stuart, M. A., Complex Coacervation Core Micelles. Colloidal Stability and Aggregation Mechanism. Langmuir 2004, 20 (4), 1073-1084. 40. van der Kooij, H. M.; Spruijt, E.; Voets, I. K.; Fokkink, R.; Cohen Stuart, M. A.; van der Gucht, J., On the Stability and Morphology of Complex Coacervate Core Micelles: From Spherical to Wormlike Micelles. Langmuir 2012, 28 (40), 14180-14191. 
For table of contents only.

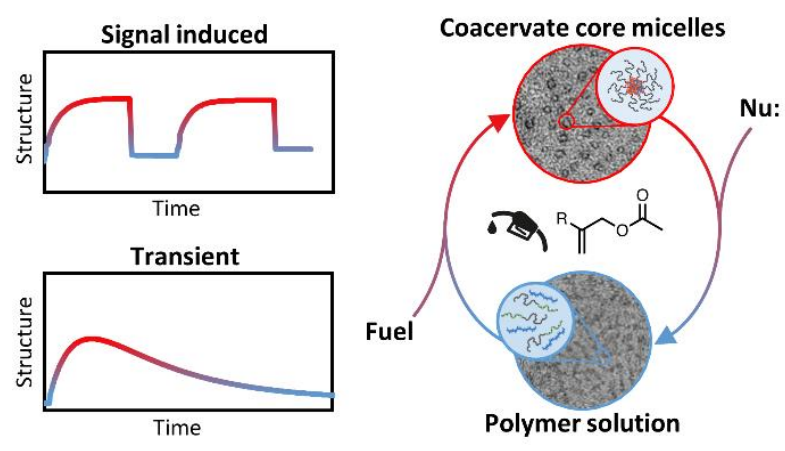

